\title{
Förderprogramm
}

\section{Lebensqualität und Lebensrealität von Patienten verbessern}

- Mit dem Förderprogramm Infektiologie, das von Gilead-Sciences ins Leben gerufen wurde, sollen Projektvorhaben unterstützt werden, die umsetzungsreif sind, deren Finanzierung jedoch nicht gesichert ist. Die eingereichten Projekte werden von einem unabhängigen Experten-Beirat anhand von definierten Kriterien bewertet. Die Fördersumme des gesamten Programms beträgt bis zu 500000 Euro pro Jahr, die sich auf erfolgversprechende Projekte in vier Kategorien verteilen:

- Klinische Kooperationsprojekte, die in Zusammenarbeit mit lokalen Organisationen in weniger entwickelten Ländern einen Beitrag zur Verbesserung der Präventi- on und/oder Therapie leisten, die Einbindung der Patienten in eine medizinische Versorgung unterstützen und deren Ziel die nachhaltige Stärkung des Gesundheitssystems in Drittländern ist.

- Forschungsprojekte, deren Ziel es ist, das Grundlagenwissen über die genannten Erkrankungen zu vertiefen. Die angestrebten Erkenntnisse sollen direkten Einfluss auf die Verbesserung der Patientenversorgung haben.

- Innovative Projekte, die die frühe Diagnose und rechtzeitige Einbindung von Patienten in eine adäquate Versorgung ihrer Erkrankung erreichen wollen. Der direkte Nutzen für Patienten, ihre Prognose und/oder ihre Lebensqualität soll im Vordergrund stehen.

- Projekte, die im Sinn der Selbsthilfe „von Betroffenen für Betroffene" einen entscheidenden Beitrag zur Versorgung der Patienten leisten, die Alltagsbedürfnisse dieser Patienten berücksichtigen und eine Verbesserung ihrer Lebensqualität erreichen wollen.

Anmeldungen zur Teilnahme an dem Programm sind ab April 2013 bis zum 15. Mai 2013 über die Webseite www.gileadfoerderprogramminfektiologie.de möglich.

- Red.

Quelle: nach Informationen der Gilead Sciences

\section{Diabetes mellitus}

\section{Hypoglykämierisiko als Limitation der Insulintherapie entschärfen}

- Rund 70\% der insulinpflichtigen Typ-2Diabetiker geben auf Befragen mindestens fünf Unregelmäßigkeiten bei der Insulintherapie im vergangenen Monat an, sei es, dass Injektionen weggelassen wurden (17\%), dass die Injektion nicht zur richtigen Zeit erfolgte (27\%) oder dass die Dosis reduziert wurde (27\%). Das dokumentiert die Erhebung GAPP2 (Global Attitudes of Patients and Physicians, Brod $M$ et al. Diabetes 2012; 61(Suppl. 1A): 27-LB, 5579), eine multinationale Onlinebefragung bei Patienten sowie bei Ärzten und auf Diabetes spezialisierten Assistenzberufen. Sehr häufig ist laut GAPP2 die Angst vor Hypoglykämien der Grund für das Abweichen von der ärztlichen Anweisung. In der Befragung gaben nach Dr. Martin Grundner, Diabetologische Schwerpunktpraxis Frankfurt, $80 \%$ der Patienten an, bereits eine Hypoglykämie erlebt zu haben.

Das Hypoglykämierisiko unter der Insulintherapie ist nach seinen Worten ein relevantes Problem: „Die Rate schwerer Unterzuckerungen liegt bei Typ-1-Diabetikern bei 0,15 bis $0,3 \%$ in Deutschland, sodass wir jährlich hierzulande mit rund 1000 schweren Unterzuckerungen bei die-

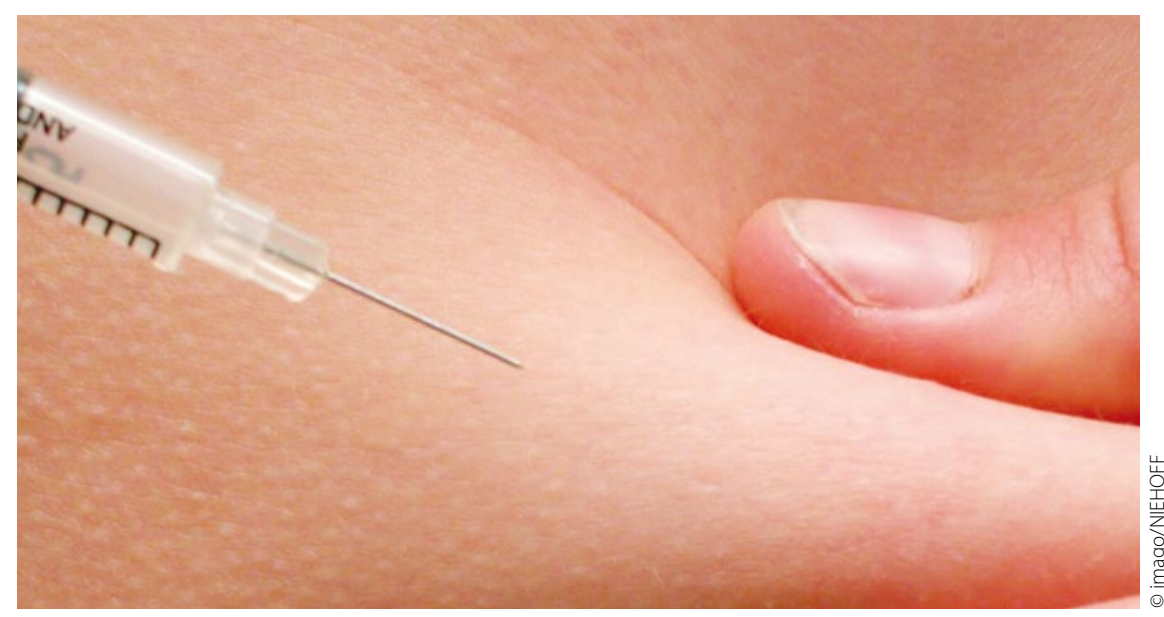

Die langsame kontinuierliche Insulinfreisetzung aus einem subkutanen Depot kann helfen, die Häufigkeit von Hypoglykämien zu senken.

sen Patienten zu rechnen haben." Auch bei Typ-2-Diabetikern sind Hypoglykämien nach seinen Worten häufiger als vermutet.

Es wird daher, so Dr. Ludwig Merker, Diabetes- und Nierenzentrum Dormagen, intensiv an der Entwicklung neuer lang wirksamer Insulinanaloga gearbeitet. Ein Vertreter dieser neuen Generation an Insulinen stellt Insulin degludec dar, bei dem durch die Injektion von löslichen Multi-Hexameren ins subkutane Gewebe eine Art
Depot entsteht, aus dem Insulin langsam und kontinuierlich in die Blutbahn diffundieren kann. Es kommt dadurch laut Merker zu einem langen und gleichmäßigen Wirkprofil und Studien zufolge zu einem um $36 \%$ reduzierten Risiko für das Auftreten nächtlicher Hypoglykämien.

\footnotetext{
- Christine Vetter

Quelle: Post-EASD Media Lunch „Latest developments in diabetes treatment", Köln, Oktober 2012 (Veranstalter: Novo Nordisk)
} 